\section{JTI}

JOURNAL OF

TRAUMA AND INJURY

\title{
Bone Transport over the Plate for the Segmental Bone Defect of Tibia
}

\author{
Il Seo, M.D. ${ }^{1,2}$, Chang-Wug Oh, M.D., Ph.D. ${ }^{1,2}$, Joon-Woo Kim, M.D., Ph.D. ${ }^{1,2}$, \\ Kyeong-Hyun Park, M.D. ${ }^{1,2}$ \\ ${ }^{1}$ Regional Trauma Center, Kyungpook National University Hospital, Daegu, Korea \\ ${ }^{2}$ Department of Orthopaedic Surgery, School of Medicine, Kyungpook National University, \\ Daegu, Korea
}

Received: June 2, 2018

Revised: July 31, 2018

Accepted: August 6, 2018

Segmental bone defects of the tibia present a challenging problem for the orthopedic trauma surgeon. These injuries are often complicated by soft tissue defects and infection. Many techniques are reported, from bone graft to bone transport. To our knowledge, bone transport over the plate in the distraction site has not been described for the treatment of tibial bone defect. We report an instance including procedure and subsequent complications after bone transport over the plate, to restore a tibial bone defect.

Keywords: Osteogenesis, Distraction; Tibia; Wounds and injuries

\section{Correspondence to}

Chang-Wug Oh, M.D., Ph.D.

Department of Orthopedic Surgery, Kyungpook National University Hospital, School of Medicine, Kyungpook National University, 130 Dongdeok-ro, Jung-gu,

Daegu 41944, Korea

Tel: +82-53-420-5630

Fax: +82-53-422-6605

E-mail:cwoh@knu.ac.kr

\section{INTRODUCTION}

Segmental bone defects of the tibia present a challenging problem for the orthopedic surgeon. The anatomy and physiology of the tibia predisposes it to healing problems, while concomitant soft tissue loss greatly increases the risk of complications. A variety of treatment strategies have been described such as bone graft, induced membrane techniques, vasculized fibula graft, as well as bone transport and bone transport over the nail. Transport over an intramedullary device has been advocated by several authors to better allow the surgeon to control length and alignment during transport, in addition to the increased rigidity of the construct, which ultimately allows for earlier removal of the external fixator [1]. However, the procedure is not foolproof. Problems with pin site infection, joint stiffness due to prolonged external fixator, and docking site nonunion can all occurr. Most authors consider prolonged external fixation, history of previous pin tract infections, and extremely proximal or distal segmental defects as contraindications for the use of intramedullary fixation [2,3]. Plate-guided bone segment transport has been described to successfully treat defects in the femur [4]. (http://creativecommons.org/licenses/by-nc/4.0/) which permits unrestricted noncommercial use, distribution, and reproduction in any medium, provided the original work is properly cited. 


\section{JTI}

We treat large tibia bone defects using locked plating and bone transport with a mono external fixation frame. This technique allows correction of length and alignment, stabilizes the limb, and facilitates earlier external fixator removal than bone transport over the nail. It also offers the opportunity to additionally compress and stabilize the transported segment at the time of docking by using additional locking screws through the plate.

\section{CASE REPORT}

A 44-year-old man experienced a crush injury to the right lower extremity resulting in a Gustilo-Anderson grade IIIB open tibia fracture with segmental bone loss (Fig. 1). Initially, he visited a local medical center where he was treated with serial debridement, with removal of all devitalized tissue, and bone fragments, and the placing of an external ring fixator. Three months after injury, the patient was referred to our hospital. Initial open wound was covered by split thickness skin graft (Fig. 2). There

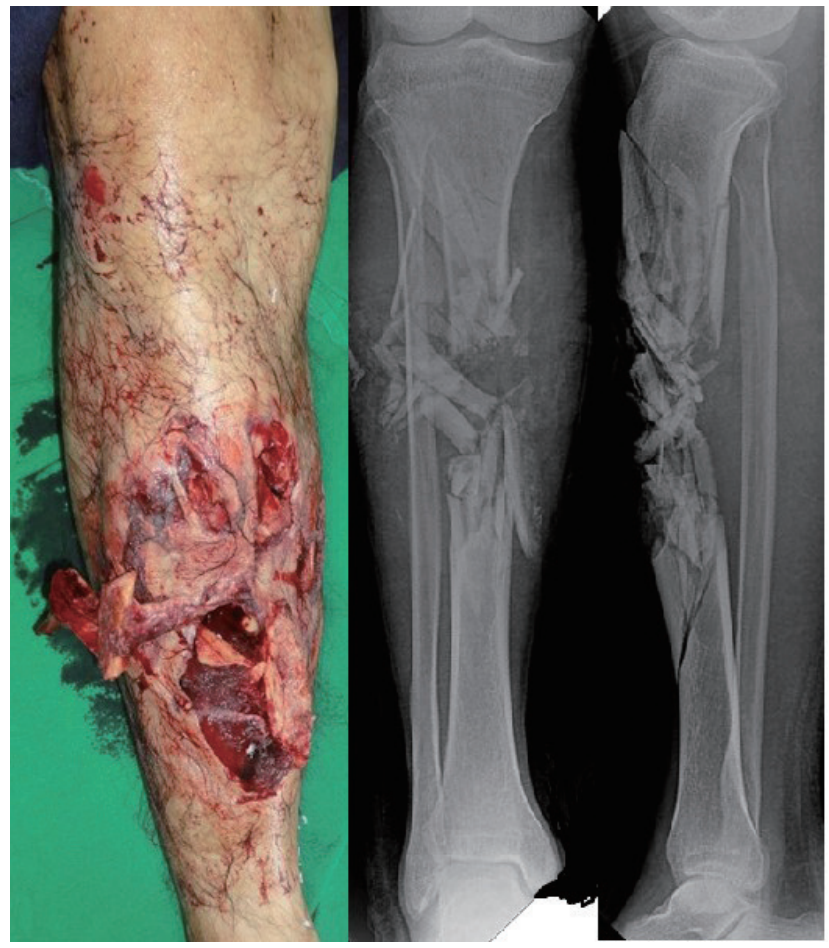

Fig. 1. 44 years old male gross photo and radio-graghs of right tibia open (Gustilo-IIIB) diaphyseal fracture. were no clinical infection signs and laboratory tests were all in normal range. Reconstruction of the tibial bone defect was undertaken using bone transport combined with minimally invasive plate osteosynthesis. The external ring fixator was removed and a monorail external fixator on anteromedial side was placed, as well as the segment of the planned corticotomy. Before corticotomy, defect site intraoperative tissue biopsy was done and shows no infection. The corticotomy was made at the middle third of his tibia (Fig. 3). Bone transport began 10 days status post corticotomy at a rate of $1 \mathrm{~mm}$ per day in four $0.25 \mathrm{~mm}$ increments. The duration of the transport period was 92 days and total time in external fixation was also 92 days. The total defect length was $86 \mathrm{~mm}$ with our ex-fix index (duration of external fixation per centimeter of bone lengthened) was $9.4 \mathrm{day} / \mathrm{cm}$. The transported segment was docked and fixed to the plate with a single percutaneous screw and autogenous bone graft from iliac crest, while the external fixator was removed simultaneously.

The reason for choosing this technique was first, the patient had already been in an external fixator for a pro-

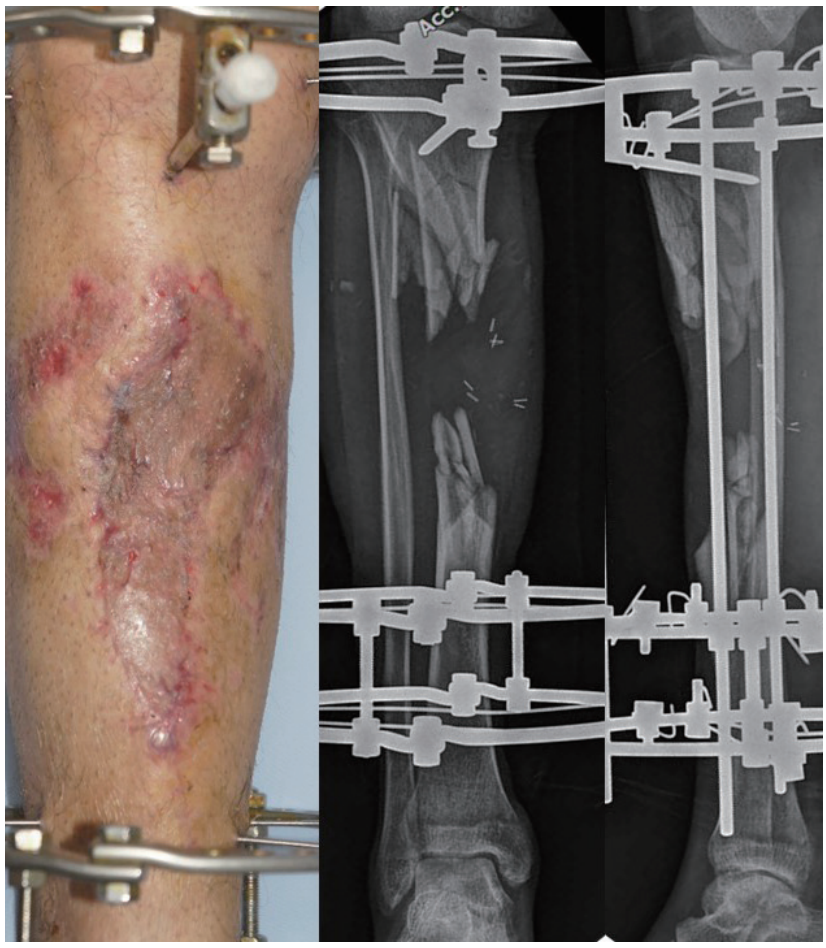

Fig. 2. Rt. tibia open fracture gross photo and X-rays at first visit our hospital. Skin defect was covered by split thickness skin graft. 


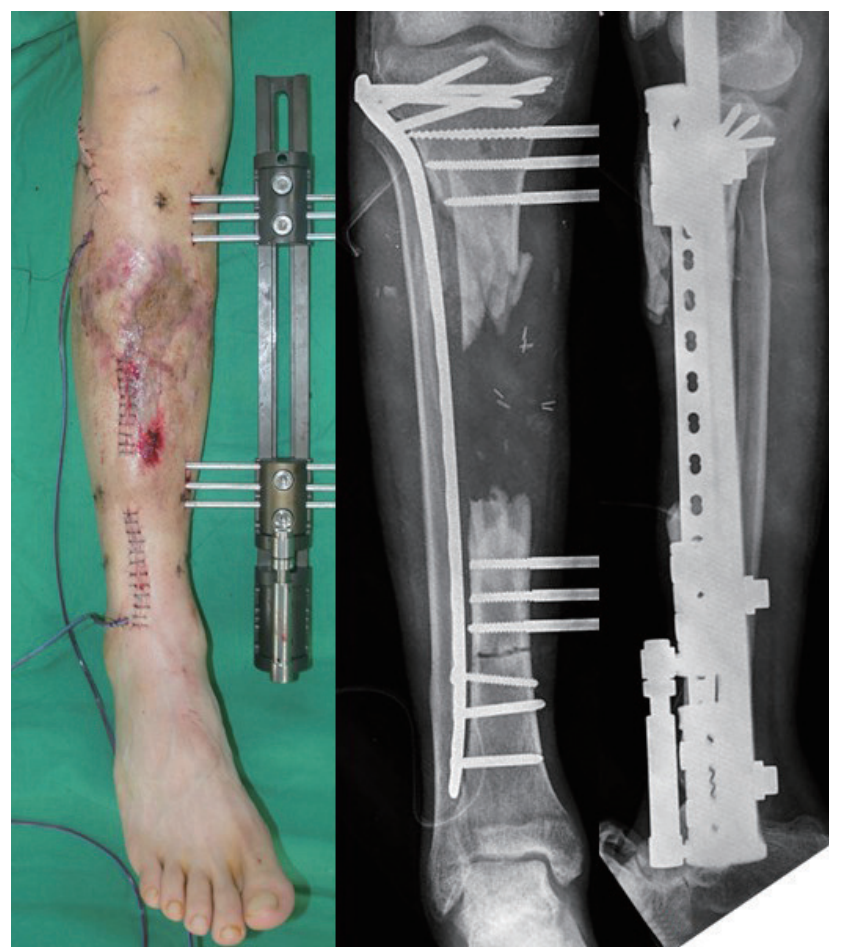

Fig. 3. Showing minimally invasive placement of $4.5 \mathrm{~mm}$ laterally based locking plate with mono frame external fixator for transport.

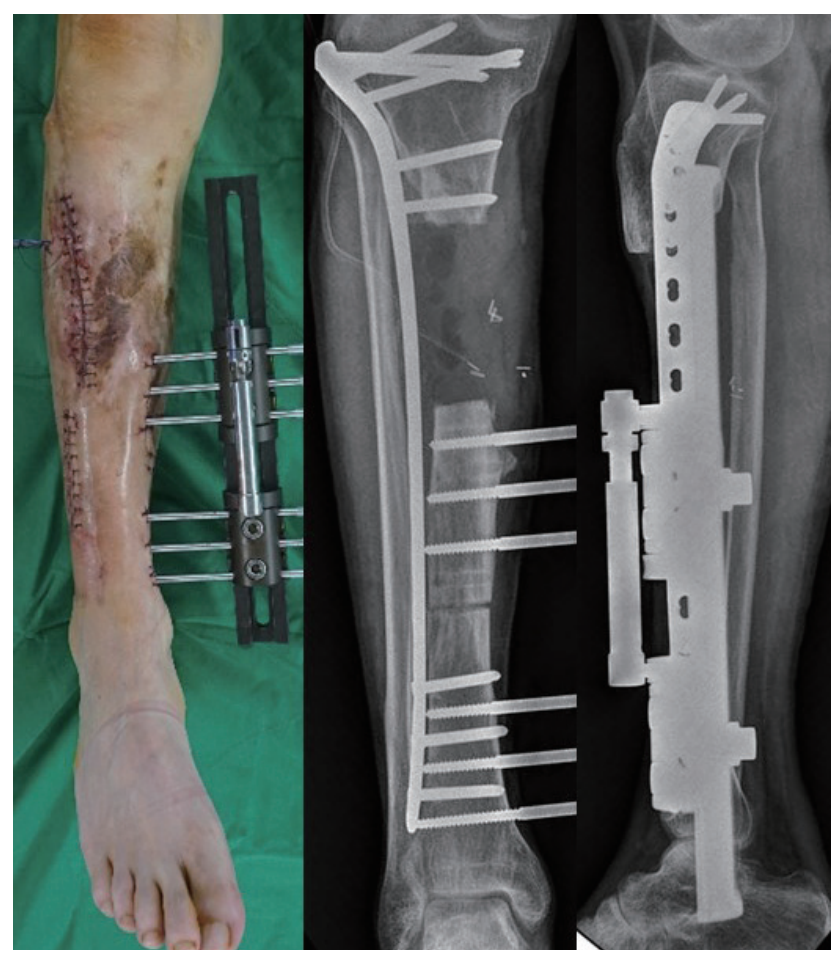

Fig. 5. Showing minimally invasive placement of $4.5 \mathrm{~mm}$ laterally based locking plate with mono frame external fixator for transport at distraction site.

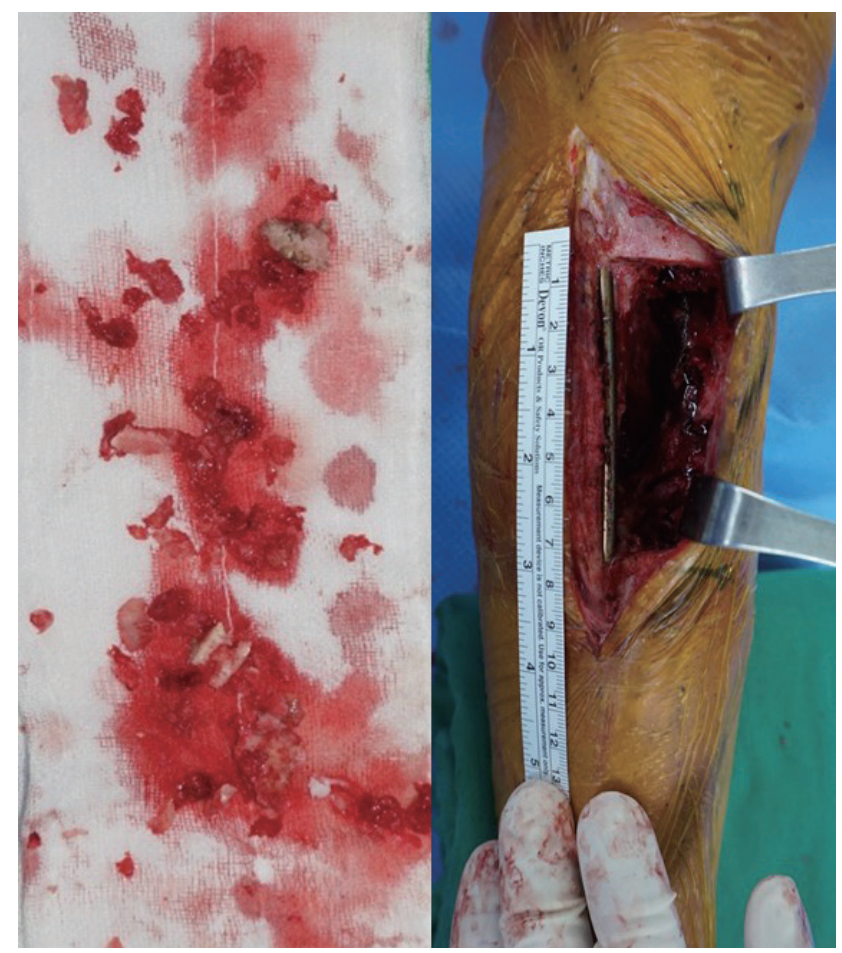

Fig. 4. Intraoperative infected tissue and post debridement defects about $70 \mathrm{~mm}$.

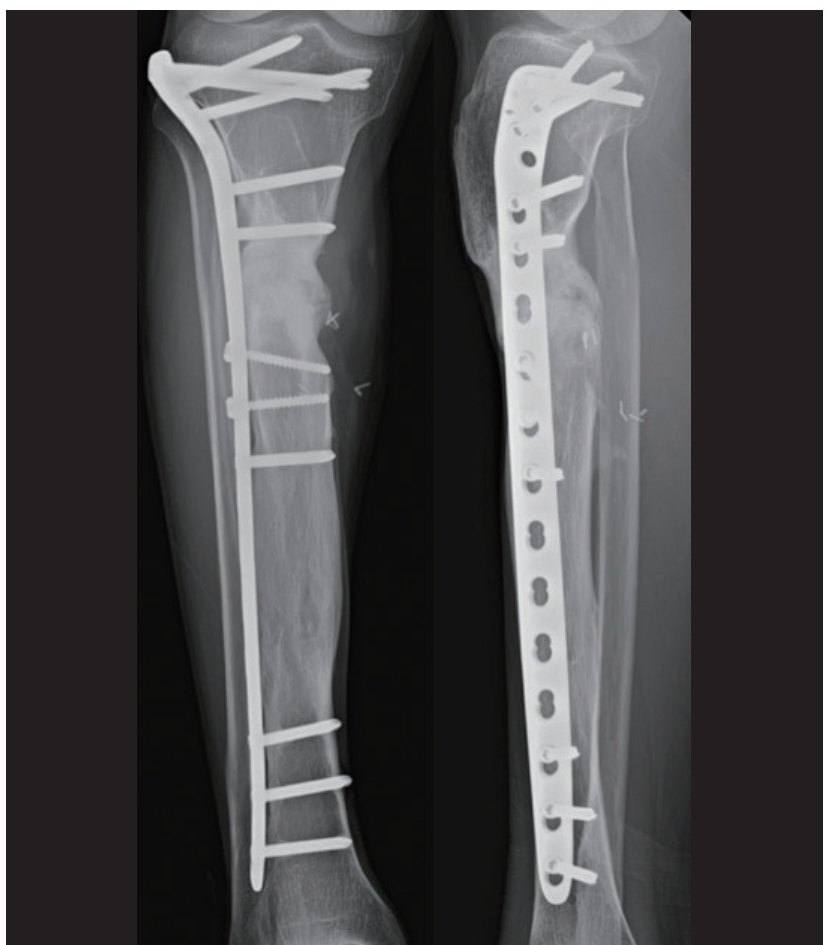

Fig. 6. Tibia radiographs showing radiographic union. 
longed period and wanted a treatment option that minimized time in a fixator. Second, long duration of external fixator pins are at risk for intramedullary sepsis if transport over an intramedullary nail was to be performed.

After 9 months complications arose. Docking site nonunion and pus discharge at the previous wound site occurred. To address these issues, bone resection around the docking site was done about $70 \mathrm{~mm}$ and anti-mixed cement beads and block were inserted (Fig. 4). Six months later, with no further infection symptoms and sign present, laboratory test and re-transport was done. Cause this patients defects was large (about $70 \mathrm{~mm}$ ) and previous large auto bone graft was done, we performed another bone transport at distraction site. The corticotomy was made at the distal tibia distraction site (Fig. 5). The duration of the second transport period was 82 days, the total defect length was $70 \mathrm{~mm}$, and our ex-fix index was 11.7 day $/ \mathrm{cm}$. After second transports, he was able to make a full weight bearing approximately 9 months from time of bone grafting. Solid radiographic union was obtained approximately 2 years from the date of transport initiation (Fig. 6). Paley-Marr functional score was good (mild limitation in ankle range of motion) and the patient was satisfied with his functional outcomes.

\section{DISCUSSION}

Tibial bone loss is a difficult problem to treat for the orthopedic trauma surgeon. External fixation with and without bone grafting, the Papineau technique, vascular pedicle grafts, induced membrane techniques, and distraction osteogenesis are all described techniques for dealing with these difficult problems. Autogenous bone grafts have been used for the management of bone defects up to 8 to $10 \mathrm{~cm}[5,6]$. However, this method requires large volumes of graft, which brings donor site problems such as pain and hematoma. For the more it takes a long time for union and weight bearing. Stress fracture and junctional nonunion is common [7].

The mean success rate for segmental bone defects treated by free vascularized bone graft is $69 \%$ and $15 \%$ of the cases required secondary surgical treatment [8]. It is worth noting that this procedure requires microsurgery techniques and long-term immobilization and bracing, which in themselves stress fracture risk at the graft site.

Masquelet et al. [9] reported a series of 35 cases of bone reconstruction of large diaphyseal defects performed in two stages. In weight bearing on diaphyseal segments normal walking was possible at 8.5 months on average.

Distraction osteogenesis, dynamic process of bone formation in which new bone forms in a bone gap through slow distraction, was suggested by Ilizarov for the treatment of segmental bone defects. It can be done for large defects greater than $10 \mathrm{~cm}$. The treatment of bone defects by callus distraction is a biological method. Advantages of this technique include the ability to correct deformity and lengthen an extremity simultaneously, eliminating donor site morbidity seen with autologous grafting techniques or free tissue transfer, the ability to treat massive defects, and the ability of the patient to bear weight with an external fixator during treatment. However, complications of this procedure include pin tract infections, broken wires, joint contractures, junctional nonunion, and malunion. Distraction osteogenesis, using Ilizarov technique, requires almost 2 months in fixation for every centimeter of defect reconstructed in a single-level transport $[10,11]$.

Bone transport using a monorail fixator over an intramedullary nail is a technique that has been used with success by multiple authors. Advantages of this technique are multiple. A statically locked intramedullary nail maintains anatomic length and alignment and ensures subsequent docking of the transported segment. The increased stability of the intramedullary device reduces the stability required by the external fixator. Interlocking of the transported segment allows protection of the regenerated callous and compression at the docking site. Finally, the external fixation device can be removed once docking has occurred, thus greatly reducing the total time a patient is required to be in a frame with lengthening indices reported of just under 1 month per centimeter lengthened [12]. However, concern for increased infection rates with this method of transport still exist if performed after a prolonged period of external fixation. In 2009, Oh et al. [13] described their method of limb lengthening using a submuscular locking plate and a unilateral external fixator. Limb lengthening was performed in 10 patients who were unsuitable for limb lengthening over an intramed- 
ullary nail. The authors' indications for lengthening using this technique were skeletal immaturity, bony deformity, narrow intramedullary canals, and joint contracture. Target length was achieved in all their patients (mean of 4.0 $\mathrm{cm}$ ) and their mean duration of external fixation was 61.6 days (45-113 days). They concluded that limb lengthening with a submuscular locking plate can successfully be used in children with anatomy not suitable for nailing [13].

Our case exhibited bone transport over the plate. This technique reduces the external fixation periods and procedural stages. But still, infection and nonunion at the docking site are major complications of this technique. In cases of segmental bone defect of the tibia where the cause is an initial high energy injury (open contaminated wound) and there is compromised soft tissue, given the nature of these injuries and despite the use of appropriate tissue transfers and other wound coverage, the risk for nonunion at the docking site is high.

In our case, where even the pin site for transport was clean, infected nonunion developed. The reason of infected nonunion may be derived from initial open contaminated wound and incomplete removal of contaminated soft tissue and bone. When performing this technique, thorough debridement of contaminated wound and a clean pin site is necessary. We advise to always be cautious about infection and to keep the patient informed of this.

In conclusion, with the bone transport over the plate method, treatment of segmental bone defects can obtain biological bone consolidation and does not require immobilization. Be aware of infection and nonunion and thorough debridement of contaminated wound and care of the pin site is needed. Corticotomy at the distraction site is one of the safest techniques in distraction osteogenesis and is one option to treat complications.

\section{REFERENCES}

1. Brunner UH, Cordey J, Kessler S, Rahn BA, Schweiberer L, Per- ren SM. Bone segment transport in combination with an intramedullary nail. Injury 1993;24 suppl 2:S29-44.

2. Blachut PA, Meek RN, O'Brien PJ. External fixation and delayed intramedullary nailing of open fractures of the tibial shaft. A sequential protocol. J Bone Joint Surg Am 1990;72:729-35.

3. Siebenrock KA, Gerich T, Jakob RP. Sequential intramedullary nailing of open tibial shaft fractures after external fixation. Arch Orthop Trauma Surg 1997;116:32-6.

4. Apivatthakakul T, Arpornchayanon O. Minimally invasive plate osteosynthesis (MIPO) combined with distraction osteogenesis in the treatment of bone defects. A new technique of bone transport: a report of two cases. Injury 2002;33:460-5.

5. Myeroff C, Archdeacon M. Autogenous bone graft: donor sites and techniques. J Bone Joint Surg Am 2011;93:2227-36.

6. Christian EP, Bosse MJ, Robb G. Reconstruction of large diaphyseal defects, without free fibular transfer, in Grade-IIIB tibial fractures. J Bone Joint Surg Am 1989;71:994-1004.

7. Prokuski LJ, Marsh L. Segmental bone deficiency after acute trauma. The role of bone transport. Orthop Clin North Am 1994;25:753-65.

8. Wei FC, El-Gammal TA, Lin CH, Ueng WN. Free fibula osteoseptocutaneous graft for reconstruction of segmental femoral shaft defects. J Trauma 1997;43:784-92.

9. Masquelet AC, Fitoussi F, Begue T, Muller GP. Reconstruction of the long bones by the induced membrane and spongy autograft. Ann Chir Plast Esthet 2000;45:346-53.

10. Green SA. Skeletal defects. A comparison of bone grafting and bone transport for segmental skeletal defects. Clin Orthop Relat Res 1994;301:111-7.

11. Paley D. Problems, obstacles, and complications of limb lengthening by the Ilizarov technique. Clin Orthop Relat Res 1990;(250):81-104.

12. Oh CW, Song HR, Roh JY, Oh JK, Min WK, Kyung HS, et al. Bone transport over an intramedullary nail for reconstruction of long bone defects in tibia. Arch Orthop Trauma Surg 2008; 128:801-8.

13. Oh CW, Song HR, Kim JW, Choi JW, Min WK, Park BC. Limb lengthening with a submuscular locking plate. J Bone Joint Surg Br 2009;91:1394-9. 